\title{
Measurements of electron beam emittance in the Accelerator Test Facility damping ring operated in multibunch modes
}

\author{
Yosuke Honda and Noboru Sasao* \\ Department of Physics, Kyoto University, Kyoto 606-8502, Japan
}

Sakae Araki, Hitoshi Hayano, Yasuo Higashi, Kiyoshi Kubo, Toshiyuki Okugi, Takashi Taniguchi, Nobuhiro Terunuma, Junji Urakawa, and Yoshio Yamazaki

High Energy Accelerator Research Organization (KEK), Ibaraki 305-0801, Japan

Koichiro Hirano, Masahiro Nomura, and Mikio Takano

National Institute of Radiological Sciences (NIRS), Chiba 263-8555, Japan

Hiroshi Sakai

Institute for Solid State Physics, University of Tokyo, Tokyo 277-8581, Japan

(Received 1 June 2003; published 24 September 2003)

\begin{abstract}
We present the measurement results of electron beam emittance in the Accelerator Test Facility damping ring operated in multibunch modes. The measurements were carried out with an upgraded laser wire beam profile monitor. The monitor has now a vertical wire as well as a horizontal one and is able to make much faster measurements thanks to an increased effective laser power inside the cavity. The measured emittance shows no large bunch-to-bunch dependence in either the horizontal or vertical directions. The values of the vertical emittance are similar to those obtained in the single-bunch operation. The present results are an important step toward the realization of a high-energy linear collider.
\end{abstract}

DOI: 10.1103/PhysRevSTAB.6.092802

PACS numbers: $41.75 . \mathrm{Ht}$

\section{INTRODUCTION}

In our previous paper [1], we reported the measurements of electron vertical emittance in the damping ring of the Accelerator Test Facility (ATF) [2], KEK, by a laser wire beam profile monitor. In these measurements, the damping ring was operated in a singlebunch mode. The target value for the vertical emittance, needed to realize a high-energy linear collider, is set to $1.1 \times 10^{-11} \mathrm{~m} \mathrm{rad}$, and it is concluded the ATF damping ring has practically achieved it at least in this operational mode.

In the present paper, we report on the emittance measurements of multibunch modes. The main motivation of this study is to confirm that similar emittance can be achieved also in the multibunch modes, and that it is independent of individual bunches. These questions are directly related to the luminosity which would be attained in colliders. Another motivation of the study is to measure horizontal emittance in the damping ring. Simultaneous measurements for both horizontal and vertical directions will lead to better understanding of low-emittance beam dynamics. To achieve this goal, we modified our setup and installed another optical cavity.

\footnotetext{
*Corresponding author.

Email address: sasao@scphys.kyoto-u.ac.jp
} wire beam profile monitor. This monitor is based on the Compton scattering of electrons with a laser wire, which is a thin light target installed in the beam. It is realized by injecting laser light into a Fabry-Perot optical cavity. The detailed description of this monitor was already given elsewhere [3] and will not be repeated. However, several improvements have been made since the last measurements; thus a brief description of the monitor will be given in the next section focusing on the changes.

The paper is organized as follows. We describe our experimental setup in the next section. In Sec. III, the experimental procedure and measurement results are presented. Section IV is devoted to discussions and conclusions.

\section{EXPERIMENTAL SETUP}

\section{A. ATF linac and damping ring}

The ATF consists of an $S$-band linac, a damping ring, and an extraction line. In this experiment, the linac was operated at $1.28 \mathrm{GeV}$. It injected up to 20 bunches at a fill into the damping ring. An initial current stored in the ring was typically $20 \mathrm{~mA}$ when 20 bunches were injected. Each bunch was about $20 \mathrm{psec}$ long and circulated the ring with the frequency of about $2 \mathrm{MHz}$. They were separated by $2.8 \mathrm{nsec}$ of each other. The bunch-to-bunch population was measured by Compton scattering count rates (see 


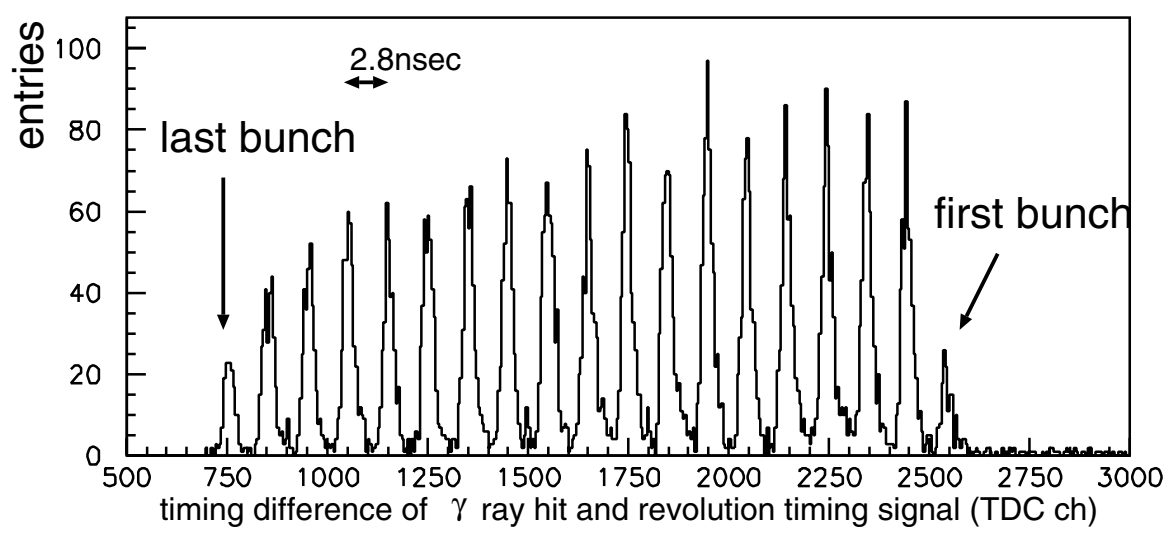

FIG. 1. Time spectrum of the gamma ray in a multibunch operation. The abscissa is the timing difference between gamma ray hits and the revolution timing signal.

Sec. IIC for the description of the detector). Figure 1 shows a time spectrum of gamma ray hits on the detector. As seen, the population was fairly uniform except for the beginning and ending few bunches.

\section{B. Laser wire}

Figure 2 shows a schematic layout of our laser wire system in use. It consists of a cw green laser, two FabryPerot optical cavities, and a photon detector (not shown in Fig. 2). The setup is installed at the north straight section of the damping ring. The laser was a diode-pumped solid state laser with a wavelength of $\lambda=532 \mathrm{~nm}$ and a nominal power of $300 \mathrm{~mW}$. We installed two cavities, one for a vertical measurement (horizontal wire) and the other for a horizontal one (vertical wire). The laser beam was switched from one cavity to the other by a combination of a Pockels cell and a polarizing beam splitter. Other important optical elements are a set of lenses to make the laser light match with the cavity's mode (mode match), an optical isolator (Faraday rotator) to prevent the light reflection back to the laser source, and $p-i-n$ photodiodes to monitor the light intensities. The whole setup was mounted on a movable table: it could be moved horizontally and vertically, and its position was monitored with an accuracy of $1 \mu \mathrm{m}$ (horizontal) and $0.2 \mu \mathrm{m}$ (vertical).

The cavity parameters are listed in Table I. The cavities were set to the fundamental $\mathrm{TEM}_{00}$ gauss mode with their length adjusted to one of the resonance peaks. Since the electron beam shape was expected to be like a thin ribbon with an aspect ratio of 10:1, the vertical laser wire used for measuring the horizontal beam size was made thicker than the horizontal one. Their beam waists were determined by measuring phase differences between the cavity's fundamental and higher modes. The waist of the horizontal wire was measured also by a new method

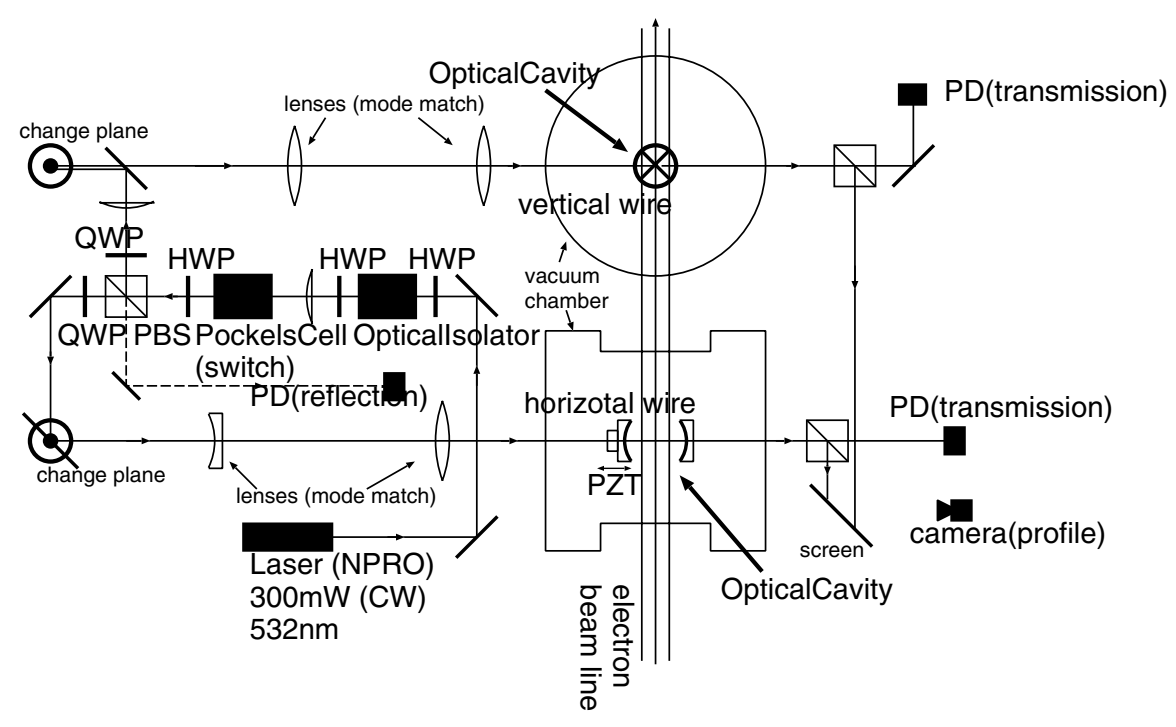

FIG. 2. Schematic layout of the laser wire setup. The symbols stand for $p-i-n$ photodiodes (PD), half-wave plate (HWP), quarterwave plate (QWP), polarizing beam splitter (PBS), and piezoactuator (PZT). 
TABLE I. Specifications of the optical cavities.

\begin{tabular}{ccc}
\hline \hline Parameter & Horizontal wire & Vertical wire \\
\hline Mirror reflectivity (front) & $99.1 \%$ & $99.8 \%$ \\
Mirror reflectivity (rear) & $99.9 \%$ & $99.9 \%$ \\
Mirror curvature & $20 \mathrm{~mm}$ & $20 \mathrm{~mm}$ \\
Finesse (measured) & 620 & 1700 \\
Power gain & 660 & 1300 \\
Effective laser power & $79 \pm 7 \mathrm{~W}$ & $156 \pm 13 \mathrm{~W}$ \\
Waist size & $11.3 \pm 0.2 \mu \mathrm{m}$ & $29.4 \pm 0.5 \mu \mathrm{m}$ \\
Rayleigh range & $760 \mu \mathrm{m}$ & $5100 \mu \mathrm{m}$ \\
Linewidth of laser & $10 \mathrm{kHz} / \mathrm{m} \mathrm{sec}^{\mathrm{a}}$ \\
\hline \hline
\end{tabular}

${ }^{\mathrm{a}}$ This means the short term (within $1 \mathrm{msec}$ ) frequency stability.

which used the electron beam itself (see Ref. [4] for details). The result by this method was found to be

$$
w_{0}=11.08 \pm 0.49(\mu \mathrm{m})
$$

which is consistent with the corresponding result in Table I. The finesse and power gain were measured by observing transmitted and reflected light from the cavity. The effective power inside the cavities was estimated to be $156 \mathrm{~W}$ for the vertical wire and $79 \mathrm{~W}$ for the horizontal (see Ref. [4] for details).

During the actual data taking, the laser effective power was modulated with the frequency of $\sim 100 \mathrm{~Hz}$. The modulation was accomplished by changing the cavity length around the resonance condition with a piezoactuator, and its amplitude was adjusted so that the lowest power was practically negligible. We defined two laser power states, "on" and "off," by observing the transmitted light from the cavity. The power off state was necessary to subtract background counts. The transmitted and reflected lights from the cavity were continuously monitored during the experiment and were found to be stable within a few $\%$.

\section{Photon detector and readout circuits}

Scattered photons were detected by a pure CsI $(70 \times$ $70 \times 300 \mathrm{~mm}^{3}$ ) crystal installed $12.7 \mathrm{~m}$ downstream of the vertical wire's cavity. The crystal was viewed by a $2 \mathrm{in}$. photomultiplier attached at the downstream end. Its energy calibration was done with passing-through cosmic rays. In order to reduce backgrounds, a lead collimator with a $5 \mathrm{~mm}$-diameter bore was placed in front of the detector. We expect the energy of the scattered photons to be in the rage of $23.0-28.6 \mathrm{MeV}$, where the high limit is given by the Compton kinematics and the low limit is determined by the collimator radius. There was a leakage of an electromagnetic shower from the side of the crystal. The actual energy deposited in the detector was 15$25 \mathrm{MeV}$.

A schematic diagram of the readout circuits is displayed in Fig. 3. The signal from the detector was first processed by an amplifier, multilevel discriminator, and logic circuits: they produced a logic pulse (NIM) only when the energy deposit was in the $15-25 \mathrm{MeV}$ range. The signal (or background as well) came synchronously with one of the bunches in the electron beam. The bunch was identified with a module called "bunch marker" (BM). This module was made with a combination of a time-toamplitude converter and a set of comparators, and had 24 outputs corresponding to each bunch. The start to the $\mathrm{BM}$ was given by an electron-revolution signal with an appropriate delay. The stop was formed by the detector logic signal. The timing resolution was dominated by time jitter of the detector signal and was estimated to be about 0.56 nsec: this should be compared with the $2.8 \mathrm{nsec}$ bunch-to-bunch spacing (see Fig. 1 for the actual performance). The output signals from the BM were fed into two 24-input scalers, one for the laser on state and the other for the off state. The scalers were enabled when the laser wire states were on or off, which in turn were determined by the $p-i-n$ photodiode signal (transmitted intensity) fed into a multilevel comparator. The actual

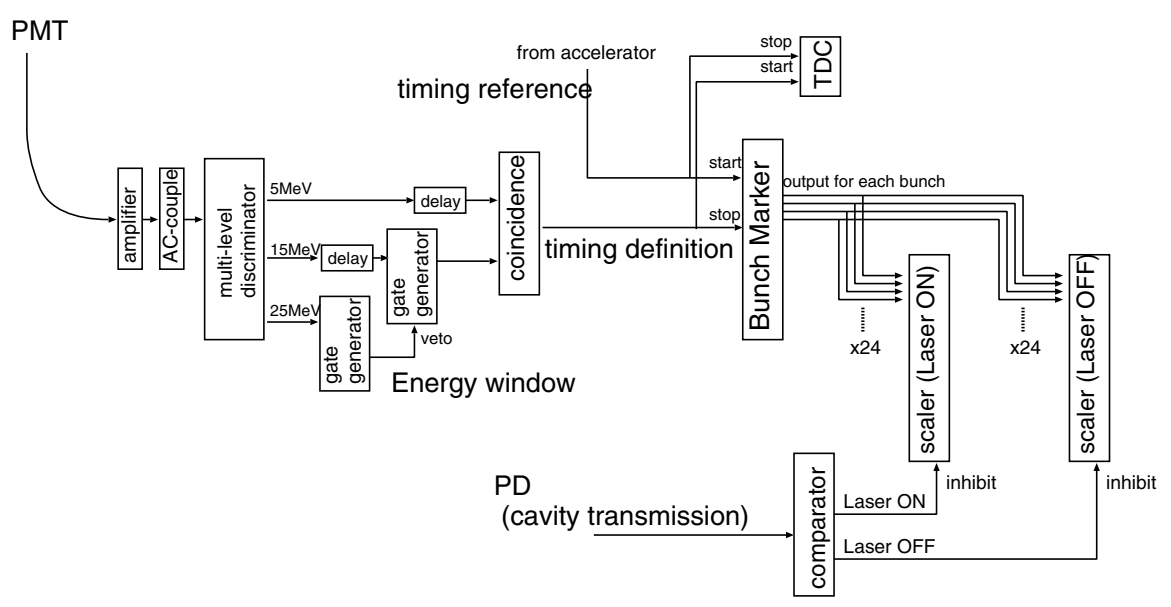

FIG. 3. Schematic diagram of the readout circuits. 
threshold levels were set in such a way that the average power of the on (off) state inside the cavity was about $85 \%(7.5 \%)$ of the maximum. The scaler data together with other relevant signals such as $p-i-n$ photodiode readings were read out by a local computer every $1 \mathrm{~s}$.

\section{Summary of improvements}

Below we summarize the important improvements and/or changes made to the previous setup. Two cavities were installed instead of one: the new one was to measure horizontal beam emittance. The old laser $(100 \mathrm{~mW})$ was replaced by a more powerful $(300 \mathrm{~mW})$ one. The power gain of the cavity was increased from 220 to 660 for the horizontal wire: this was achieved by employing a mirror with higher reflectivity. We note that the power gain of 1300 was achieved for the vertical wire. The readout circuits were modified to handle the multibunch data. A whole scan of the electron beam was computer controlled and automated. With these improvements, the complete scan (to obtain one value of the electron beam size) took about $6 \mathrm{~min}$ for the vertical measurement and $15 \mathrm{~min}$ for the horizontal.

Thanks to the increase in the laser effective power, the signal-to-noise ratio was also improved. Figure 4 shows the energy spectra of detected photons with (a) the laser wire on and (b) off states when the laser wire was placed on the beam center. A clear signal can be seen in the expected energy range. The vertical lines in the figure show the window of our signal region. The signal-to-noise ratio was 3:1 for the horizontal wire and 1:3 for the vertical.

\section{EXPERIMENT AND RESULTS}

\section{A. Data and data taking procedure}

Data were taken for two different multibunch modes: in one mode 20 bunches and in the other 3 bunches were injected in the ring. In both cases, the scan was made both horizontally and vertically. Thus four different sets of the data were obtained in total.

One run was defined to be one complete scan of a round-trip from one laser wire position to another, and
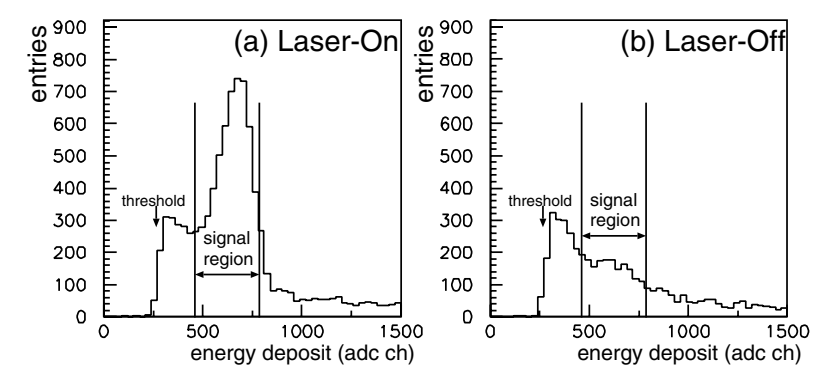

FIG. 4. Energy spectra measured by the CsI detector. (a) The laser "on" state; (b) "off" state. back to the original position. The scan was either horizontal or vertical, and it covered a whole electron beam profile. Actually, the data taking time was 10 -s long per point at every $10 \mu \mathrm{m}$ for the vertical scan and 30-s long at every $50 \mu \mathrm{m}$ for the horizontal. The electron current in the ring decreased during the scan: thus a new fill was sometimes needed especially during high-current measurements. Thus data of one complete scan contained several fills $(2 \sim 10)$ for high-current data and only one fill for low-current data.

Raw scaler data were first classified according to the laser wire position, the bunch identification, and the electron beam current. The data were normalized to a counting rate per unit time and unit current $(\mathrm{Hz} / \mathrm{mA})$, and then the laser off rate was subtracted from the laser on rate. The resultant value, signal count rate, was then plotted as a function of the laser wire position. Figure 5 shows an example of the signal count rate, in which the solid line is a Gaussian fit to the data points. From this fit, we extract three quantities: the height (the maximum of the signal count rate), the width (the measured beamwidth $\left.\sigma_{\text {meas }}\right)$, and the central position $\left(x_{p}, y_{p}\right)$. Among those, $\sigma_{\text {meas }}$ is of our main interest, but the other two quantities are also useful to monitor the stability of the beam.

\section{B. Bunch dependence}

We first show the results which test whether or not bunch-to-bunch dependence exists in the measured electron beam size. Figure 6 shows an example of such results for the vertical measurement. These particular data correspond to the 20-bunch beam of $I=18 \mathrm{~mA}$. The ordinates are (a) the peak height, (b) the peak position $\left(y_{p}\right)$, and $(\mathrm{c})$ the peak width $\left(\sigma_{\text {meas }}^{(y)}\right)$, and the abscissa is the bunch number. Figures 7-9 show the corresponding results for the 20-bunch horizontal, the 3-bunch vertical, and the 3-bunch horizontal measurements, respectively. As can be seen, there is no large bunch-to-bunch dependence either in the peak position or the width.

We checked all other data at different currents and concluded that there was no significant bunch dependence. In order to quantify the statement above, we calculated
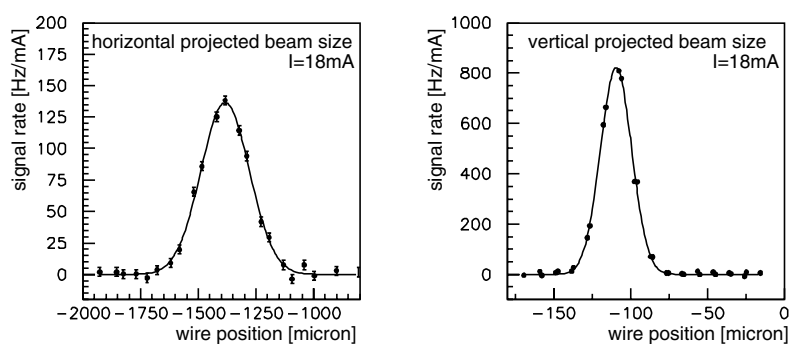

FIG. 5. Signal counts vs the laser wire position. (Left panel) horizontal scan; (right panel) vertical scan. 


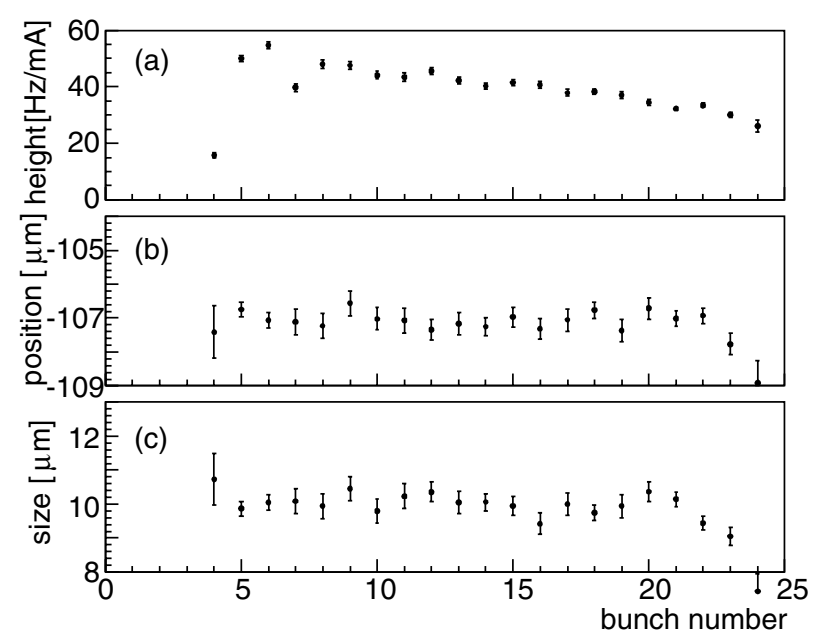

FIG. 6. Bunch dependence of the 20-bunch beam (vertical measurement). (a) The peak height; (b) the vertical peak position $y_{p} ;$ (c) the peak width $\sigma_{\text {meas }}^{(y)}$.

the average of $\sigma_{\text {meas }}$ weighted by its error, and the error of the average itself. The results are listed in the 4th and 5th columns of Table II. The 6 th and 7 th columns are the chi square per degree of freedom $\left(\chi^{2} / \nu\right)$ and the rescaled error, i.e., $\Delta \sigma_{\text {bunch }}=\left\langle\delta \sigma_{\text {meas }}\right\rangle \sqrt{\chi^{2} / \nu}$. We consider the bigger of $\left\langle\delta \sigma_{\text {meas }}\right\rangle$ and $\Delta \sigma_{\text {bunch }}$ to be a measure of the bunch-to-bunch variation in $\sigma_{\text {meas }}$. As can be seen, the fractional variations are about $5 \%$ for the vertical and about $10 \%$ for the horizontal.

\section{Current dependence}

Next, we consider the current dependence of emittance. Here we combine the data from all different bunches together. The measured beam size $\sigma_{\text {meas }}$ and the beam emittance $\varepsilon$ are related to each other by

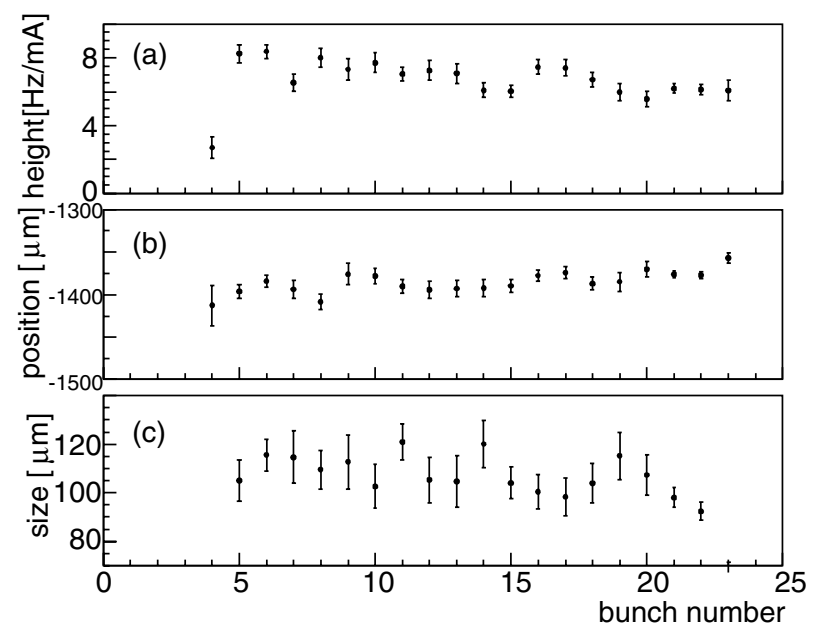

FIG. 7. Bunch dependence of the 20-bunch beam (horizontal measurement). (a) The peak height; (b) the horizontal peak position $x_{p}$; (c) the peak width $\sigma_{\text {meas }}^{(x)}$.

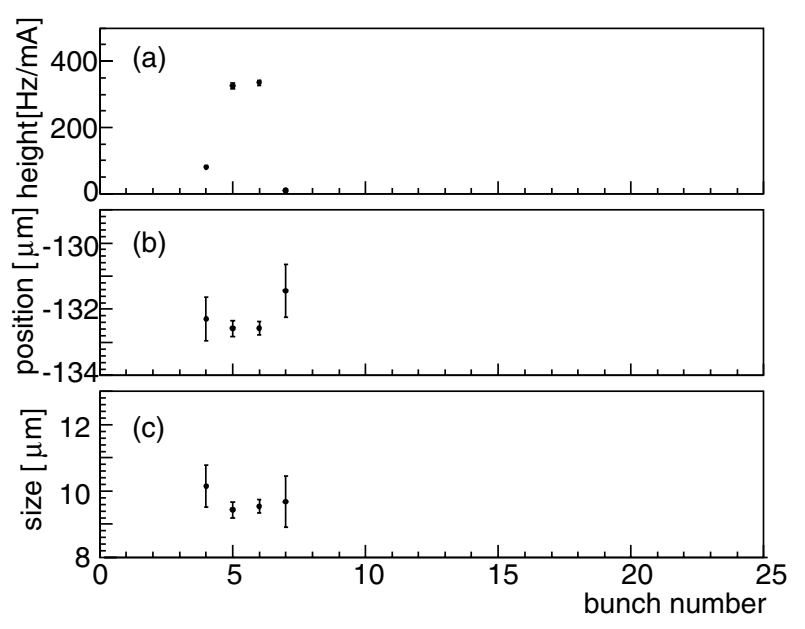

FIG. 8. Bunch dependence of the 3-bunch beam (vertical measurement). (a) The peak height; (b) the vertical peak position $y_{p}$; (c) the peak width $\sigma_{\text {meas }}^{(y)}$.

$$
\begin{gathered}
\sigma_{\text {meas }}=\sqrt{\left(\sigma_{\text {beam }}\right)^{2}+\left(w_{0} / 2\right)^{2}}, \\
\sigma_{\text {beam }}=\sqrt{\beta \varepsilon+\left(\eta \frac{\sigma_{p}}{p}\right)^{2}},
\end{gathered}
$$

where $\beta$ and $\eta$ are the beta and dispersion functions of the beam, and $\sigma_{\text {beam }}$ and $\sigma_{p} / p$ are the electron beamwidth and fractional momentum spread, respectively. The expressions above hold for both horizontal $(x)$ and vertical $(y)$ directions, separately. In practice, the dispersion term $\eta\left(\sigma_{p} / p\right)$ can be neglected because it is small. The measurement method of the $\beta$ function is the same as in the previous experiment (see Ref. [1]), and the obtained results are summarized in Table III. From Eq. (3) and $\beta$

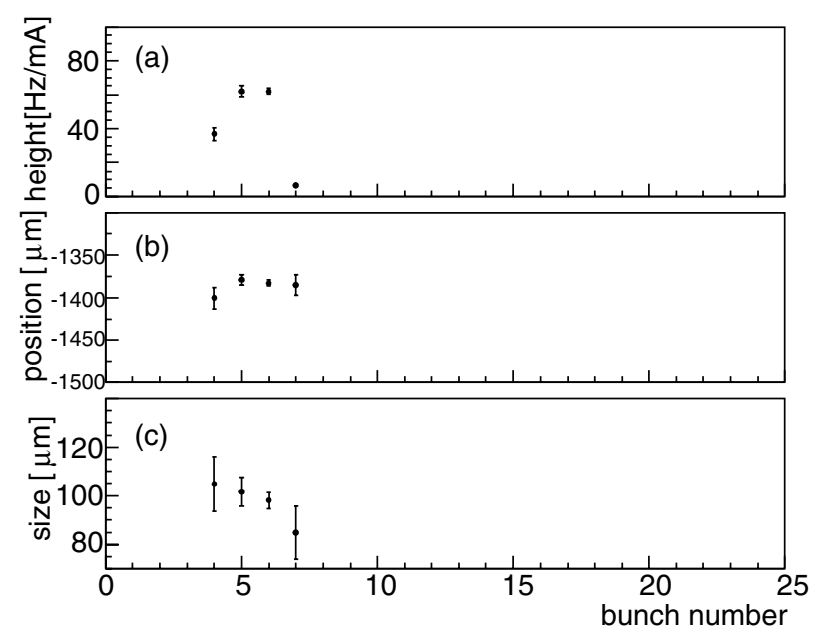

FIG. 9. Bunch dependence of the 3-bunch beam (horizontal measurement). (a) The peak height; (b) the horizontal peak position $x_{p}$; (c) the peak width $\sigma_{\text {meas }}^{(x)}$. 
TABLE II. Summary of bunch dependence of the beamwidth $\sigma_{\text {meas }}$.

\begin{tabular}{|c|c|c|c|c|c|c|}
\hline Bunch & Current & Direction & $\left\langle\sigma_{\text {meas }}\right\rangle$ & $\left\langle\delta \sigma_{\text {meas }}\right\rangle$ & $\chi^{2} / \nu$ & $\Delta \sigma_{\text {bunch }}$ \\
\hline \multirow[t]{2}{*}{20} & \multirow[t]{2}{*}{$2 \mathrm{~mA}$} & Horizontal & 89.8 & 10.14 & $17.03 / 17$ & $10.15 \mu \mathrm{m}$ \\
\hline & & Vertical & 8.716 & 0.40 & $14.38 / 17$ & $0.370 \mu \mathrm{m}$ \\
\hline \multirow[t]{2}{*}{20} & \multirow[t]{2}{*}{$5 \mathrm{~mA}$} & Horizontal & 94.4 & 9.62 & $13.36 / 17$ & $8.53 \mu \mathrm{m}$ \\
\hline & & Vertical & 9.16 & 0.36 & $22.08 / 17$ & $0.409 \mu \mathrm{m}$ \\
\hline \multirow[t]{2}{*}{20} & \multirow[t]{2}{*}{$10 \mathrm{~mA}$} & Horizontal & 100.1 & 7.85 & $10.95 / 17$ & $6.30 \mu \mathrm{m}$ \\
\hline & & Vertical & 9.45 & 0.37 & $23.25 / 17$ & $0.437 \mu \mathrm{m}$ \\
\hline \multirow[t]{3}{*}{20} & \multirow[t]{3}{*}{$18 \mathrm{~mA}$} & Horizontal & 104.5 & 8.16 & $26.83 / 17$ & $10.25 \mu \mathrm{m}$ \\
\hline & & Vertical & 9.95 & 0.30 & $18.21 / 17$ & $0.310 \mu \mathrm{m}$ \\
\hline & & Vertical & 9.94 & 0.20 & $53.55 / 17$ & $0.350 \mu \mathrm{m}$ \\
\hline \multirow[t]{2}{*}{3} & \multirow[t]{2}{*}{$0.5 \mathrm{~mA}$} & Horizontal & 102.5 & 8.84 & $0.96 / 2$ & $6.09 \mu \mathrm{m}$ \\
\hline & & Vertical & 9.41 & 0.33 & $0.857 / 2$ & $0.216 \mu \mathrm{m}$ \\
\hline \multirow[t]{2}{*}{3} & \multirow[t]{2}{*}{$1 \mathrm{~mA}$} & Horizontal & 99.41 & 6.78 & $0.53 / 2$ & $3.49 \mu \mathrm{m}$ \\
\hline & & Vertical & 8.70 & 0.28 & $6.37 / 2$ & $0.508 \mu \mathrm{m}$ \\
\hline \multirow[t]{2}{*}{3} & \multirow[t]{2}{*}{$3 \mathrm{~mA}$} & Horizontal & 99.19 & 6.75 & $1.33 / 2$ & $5.50 \mu \mathrm{m}$ \\
\hline & & Vertical & 9.53 & 0.35 & $1.12 / 2$ & $0.26 \mu \mathrm{m}$ \\
\hline
\end{tabular}

TABLE III. Summary of $\beta$ function and dispersion measurements.

\begin{tabular}{lccc}
\hline \hline Collision point & Direction & $\beta$ function $(\mathrm{m})$ & Dispersion $(\mathrm{mm})$ \\
\hline Horizontal wire & $x$ & $9.81 \pm 1.25$ & N/A \\
& $y$ & $4.32 \pm 0.20$ & $1.83 \pm 0.31$ \\
Vertical wire & $x$ & $7.83 \pm 1.25$ & $2.00 \pm 0.66$ \\
& $y$ & $4.89 \pm 0.20$ & N/A \\
\hline \hline
\end{tabular}

functions, we calculate the emittance values: the results are shown in Figs. 10 and 11 for the 20-bunch beam, and in Figs. 12 and 13 for the 3-bunch beam, respectively.

Several comments are appropriate here. First of all, the observed vertical emittance is in the range of (1-2) $\times$ $10^{-11} \mathrm{~m}$ rad: this value is somewhat larger than the target value of $1.1 \times 10^{-11} \mathrm{~m} \mathrm{rad}$, but it certainly is a very

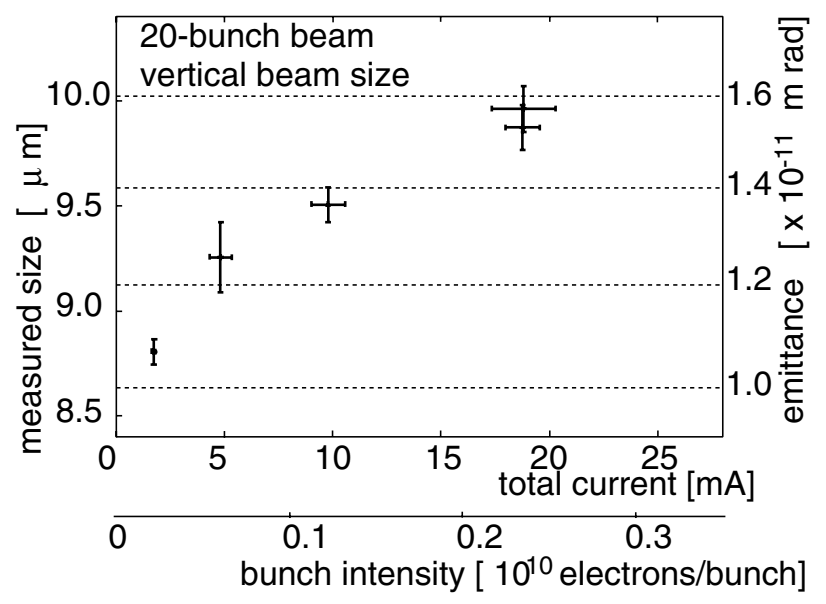

FIG. 10. Vertical emittance of the 20-bunch beam vs the electron current.

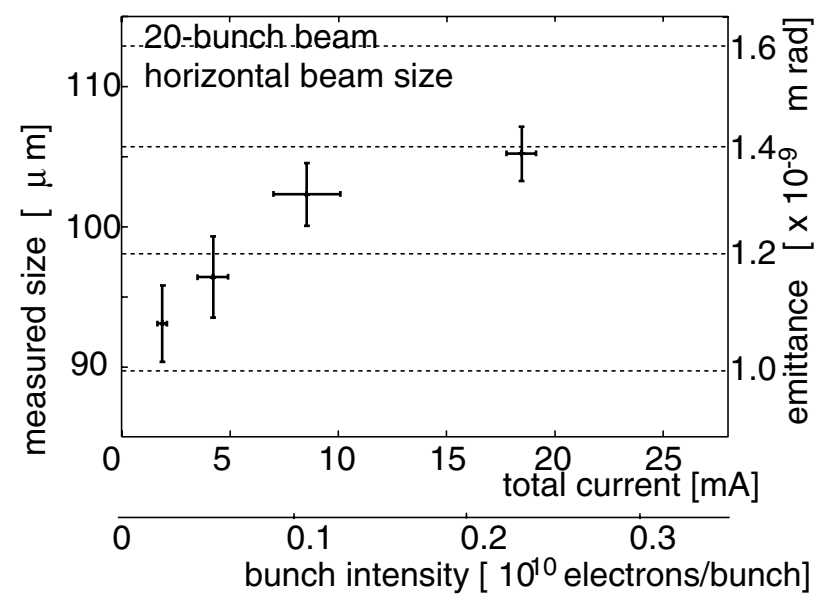

FIG. 11. Horizontal emittance of the 20-bunch beam vs the electron current.

promising result toward the realization of linear collider projects, given the fact that these are the first measurements of this kind. Second, the ratio of horizontal to vertical emittance is found to be about 100:1. This fact indicates the smallness of the horizontal and vertical coupling effects, as anticipated. Finally, as to the current dependence of the emittance, we obtained somewhat contradictory results. As seen in Figs. 10-13, there seems to exist clear current dependence in all but the 3-bunch vertical data. We checked possible systematic effects in that data (Fig. 12) and found no obvious source. ${ }^{2}$ We

\footnotetext{
${ }^{2}$ We expect the main source to be the beam orbit drift during the measurement. Other possible sources are bunch-to-bunch dependence of the beam central position and/or energy. However, there was no evidence in the data large enough to prove such effects.
} 


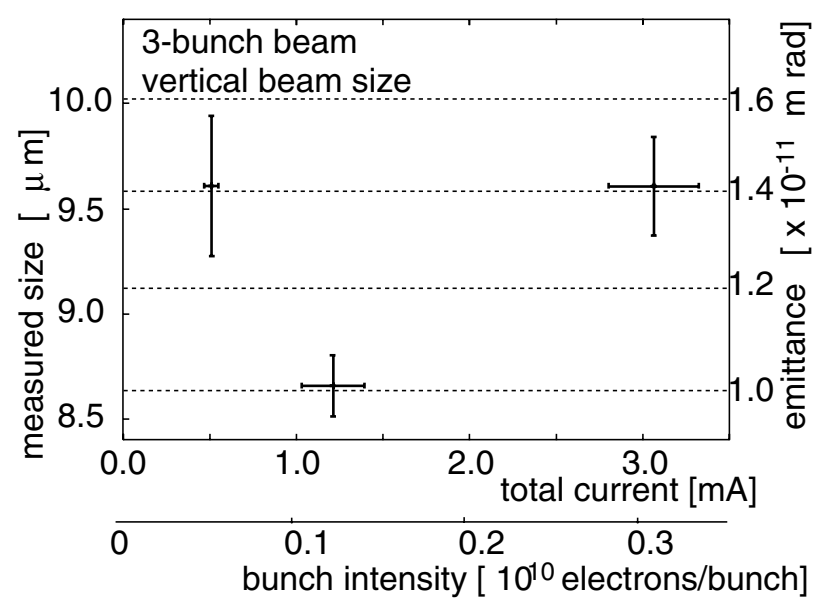

FIG. 12. Vertical emittance of the 3-bunch beam vs the electron current.

conclude that further measurements are needed to clarify the situation.

Next we want to compare the present results with the result of Ref. [1] which measured the vertical emittance in the single-bunch mode. For convenience, the result is replotted in Fig. 14 as a function of the bunch population. We observe that, if the data points in the similar bunch population are compared, the measured values agree fairly well each other. ${ }^{3}$

\section{DISCUSSIONS AND CONCLUSIONS}

In this paper, we have presented the measurements of multibunch electron beam emittance in the ATF damping ring. The measurements were carried out by the laser wire beam profile monitor. Improvements made to the previous setup were, among others, installation of the vertical laser wire and increase in the laser effective power inside the cavity. The latter enabled us much faster data taking with better precision.

The measurement results show that there is no large bunch-to-bunch dependence in the emittance. As to the current dependence of the emittance, although there is an indication of the current dependence, we believe that a definite conclusion is yet to be reached. The values of the vertical emittance in the multibunch mode are in good agreement with those in the single bunch at the corresponding bunch population. In conclusion, the results presented in this paper show an encouraging step toward the realization of future linear colliders. Finally, we would like to stress the importance of further studies both in the single and multibunch modes with better accuracy and in a wider current range.

\footnotetext{
${ }^{3}$ In our previous experiment, the effective laser intensity was 1 order of magnitude lower. This improvement drastically reduced the statistical error.
}

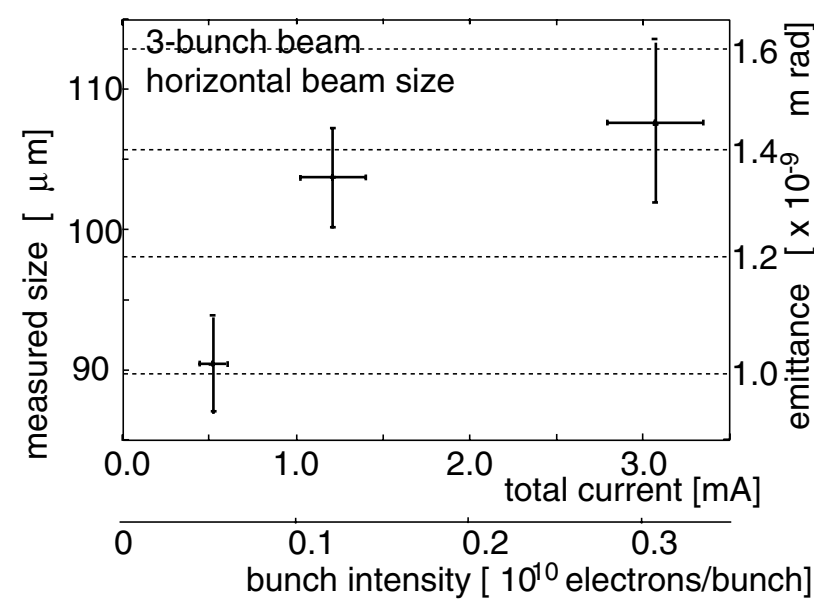

FIG. 13. Horizontal emittance of the 3-bunch beam vs the electron current.

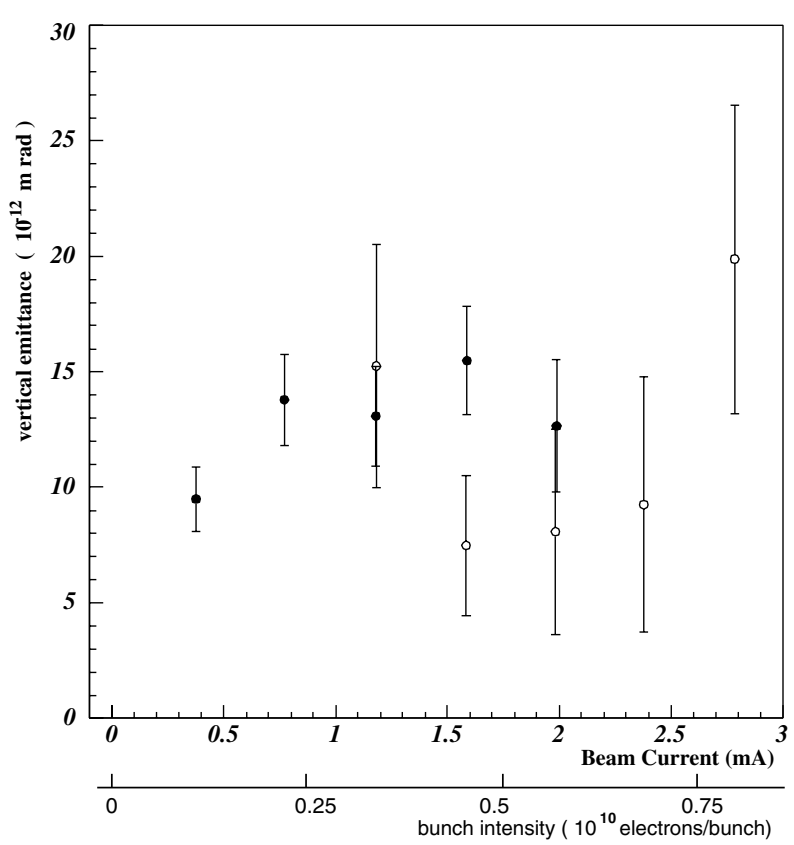

FIG. 14. Vertical emittance in the single-bunch mode (from Ref. [1]).

Together with a better monitor and control on various ring parameters, such studies will shed light on the electron beam dynamics in the ultralow emittance regime.

\section{ACKNOWLEDGMENTS}

We would like to thank all members of the ATF group for their helpful support. We are also grateful to Dr. M. Ross and Dr. J. Frisch for their useful discussions with our laser wire experiment. It is our pleasure to thank Professor H. Sugawara, Professor M. Kihara, Professor Y. Kimura, Professor S. Iwata, and Professor K. Takata for 
their support and encouragement. This research was partially supported by Grant-in-Aid Scientific Research (1344078) from the Ministry of Education, Science, Sports and Culture of Japan and the Advanced Compact Accelerator Project of the National Institute of Radiological Science.
[1] H. Sakai et al., Phys. Rev. ST Accel. Beams 5, 122801 (2002).

[2] KEK Internal Report No. 95-4, 1995, edited by F. Hinode et al.

[3] H. Sakai et al., Jpn. J. Appl. Phys. 41, 6398 (2002).

[4] Y. Honda et al. (to be published). 